\title{
Percepção de enfermeiros sobre diagnóstico e acompanhamento de mulheres com depressão pós-parto
}

RESUMO | Objetivo: Analisar as percepções de enfermeiros sobre diagnóstico e acompanhamento da depressão pós-parto em Divinópolis-MG. Método: Estudo qualitativo, descritivo, realizado no período de setembro de 2018 através de roteiro semiestruturado e referencial Minayo, com amostra de nove enfermeiros da Estratégia de saúde da família. Resultados: Os enfermeiros não possuem suporte literário pré-definido para seguir caso deparem com mulheres em depressão pós-parto, sendo essas direcionadas para o psicólogo ou psiquiatra. Nas unidades não existem capacitação para os profissionais relacionados à temática, impactando negativamente nos atendimentos, tornando-o fragmentado. Não há um assessoramento por parte do município para auxiliar os profissionais de enfermagem a lidarem com essas mulheres. São utilizados mecanismos relacionados a busca ativa na maioria das unidades do estudo. Conclusão: É de suma importância o assessoramento municipal diretamente relacionado a temática, uma vez que contribui para um atendimento integral que vai de acordo com as diretrizes do Sistema Único de Saúde.

Palavras-chaves: Atenção primária à saúde; Depressão; Enfermagem; Período pós-parto; Saúde pública.

ABSTRACT | Objective: To analyze nurses' perceptions about the diagnosis and monitoring of postpartum depression in Divinópolis-MG. Method: Qualitative, descriptive study, carried out in the period of September 2018 through a semi-structured and referential Minayo script, with a sample of nine nurses from the Family Health Strategy. Results: Nurses do not have predefined literary support to follow if they encounter women in postpartum depression, and these are directed to the psychologist or psychiatrist. In the units, there is no training for professionals related to the theme, negatively impacting the service, making it fragmented. There is no advice from the municipality to assist nursing professionals in dealing with these women. Mechanisms related to active search are used in most units of the study. Conclusion: Municipal advice directly related to the topic is extremely important, since it contributes to comprehensive care that goes according to the guidelines of the Unified Health System.

Keywords: Primary health care; Depression; Nursing; Postpartum period; Public health.

RESUMEN I Objetivo: analizar las percepciones de las enfermeras sobre el diagnóstico y seguimiento de la depresión posparto en Divinópolis-MG. Método: Estudio cualitativo, descriptivo, realizado en el período de septiembre de 2018 a través de un guión de Minayo semiestructurado y referencial, con una muestra de nueve enfermeras de la Estrategia de Salud Familiar. Resultados: Las enfermeras no tienen un apoyo literario predefinido a seguir si se encuentran con mujeres en depresión posparto, y estas están dirigidas al psicólogo o psiquiatra. En las unidades, no hay capacitación para profesionales relacionados con el tema, lo que impacta negativamente en el servicio y lo fragmenta. No hay consejos del municipio para ayudar a los profesionales de enfermería a tratar con estas mujeres. Los mecanismos relacionados con la búsqueda activa se utilizan en la mayoría de las unidades del estudio. Conclusión: El asesoramiento municipal directamente relacionado con el tema es extremadamente importante, ya que contribuye a una atención integral que se ajusta a las pautas del Sistema Único de Salud.

Palabras claves: Atención primaria de salud; Depresión; Enfermería; Período posparto; Salud pública.

\section{Flavia Karen dos Santos}

Enfermeira. Graduação em Enfermagem pela Universidade de Itaúna (UIT), Minas Gerais. ORCID: 0000-0002-1205-3009

\section{Samara Cristina da Silva}

Graduanda em Enfermagem pela Universidade do Estado de Minas Gerais (UEMG), Unidade Divinópolis.

ORCID: 0000-0003-4032-5259

\section{Marla Ariana Silva}

Graduanda em Enfermagem pela Universidade do Estado de Minas Gerais (UEMG), Unidade Divinópolis.

ORCID: 0000-0003-0136-7122

\section{Karen dos Santos Lago}

Graduanda em Enfermagem pela Universidade do Estado de Minas Gerais (UEMG), Unidade Divinópolis.

ORCID: 0000-0002-4502-5098

\section{Silmara Nunes Andrade}

Enfermeira. Doutora em Ciências da Saúde pela Universidade Federal de São João Del Rei (UFSJ). Docente do Curso de Graduação em Enfermagem da Universidade do Estado de Minas Gerais (UEMG), Unidade Divinópolis. ORCID: 0000-0002-1975-0827

Recebido em: 23/07/2020 Aprovado em: 19/08/2020

\section{Regina Consolação dos Santos}

Enfermeira. Mestre em Ciência pela Universidade Federal de São João Del Rei, (UFSJ). Docente do Curso de Graduação em Enfermagem da Universidade do Estado de Minas Gerais (UEMG), Unidade Divinópolis.

ORCID: 0000-0002-7393-3210

INTRODUÇÃO

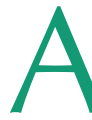
Atenção Primária à Saúde (APS) foi criada para servir como porta de entrada aos serviços do Sistema Único de Saúde (SUS). Um estudo aponta que $80 \%$ dos 
casos da atenção secundária e terciária poderiam ter sido evitados, caso o usuário tivesse se dirigido a APS. A gestão desse nível de complexidade deve incorporar em qualquer uma de suas classificações a prevenção de doenças, promoção da saúde e reabilitação, buscando a integralidade da atenção ao usuário, melhoria da satisfação, e vínculo com o indivíduo que busca o serviço ${ }^{(1)}$. Faz-se necessário uma equipe multiprofissional para se chegar à integralidade da assistência, a busca para que não haja uma atenção parcial ao assessoramento da população deve ser contínua ${ }^{(2)}$.

Dentre os desafios da atenção básica encontra-se a depressão pós-parto (DPP), que pode afetar a saúde materna até o segundo ano do bebê, e tem vínculo com a APS, desde o seu diagnóstico, acompanhamento e alta ${ }^{(3)}$. Quando se fala em funções da enfermagem sobre a DPP, espera-se minimamente, conhecimento do profissional quanto à patologia e gestão da unidade. A ESF, se bem organizada, consegue fazer a busca ativa dessas mulheres para prevenção e acompanhamento eficaz, uma vez que detectada precocemente e realizado o acompanhamento pela equipe multidisciplinar, pode-se evitar maiores complicações, como por exemplo, óbitos ${ }^{(4)}$.

A mulher começa a se culpar por coisas que fogem do padrão esperado, se sente ansiosa e preocupada sem razão aparente, além de se sentir muito atarefada em relação a casa e ao bebê. Isso mostra a necessidade de um melhor assessoramento governamental sobre puérperas, pois além dos prejuízos já existentes nessas mulheres, seus filhos também entram nos fatores de risco quando se fala em: nutrição, desenvolvimento e crescimento, emocional, e doenças diarreicas ${ }^{(5)}$.

Os sintomas são vegetativos, cognitivos e psicomotores, como alterações no humor, no apetite, no sono, irritabilidade, crises de choro, falta de concentração e energia, fadiga e desinteresse em atividades que antes gostava de fazer. Podem ocorrer também pensamentos de suicídio e culpa vindo a ocorrer desde as primeiras semanas após o parto, como a qualquer momento do primeiro ano de vida da criança $^{(6)}$. Podendo ser os fatores de risco para algumas mulheres: fatores demográficos e econômicos, multiparidade, falta de apoio e dificuldades de cumprir as tarefas normais que antes eram simples e que se tornaram difíceis devido à sobrecarga ${ }^{(7-8)}$.
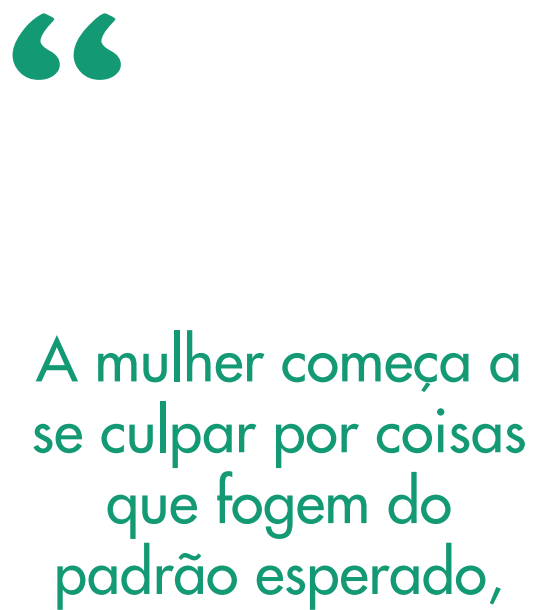
se sente ansiosa e preocupada sem razão aparente, além de se sentir muito atarefada em relação a casa e ao bebê.

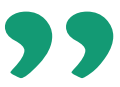

O bom relacionamento conjugal e familiar é considerado fator protetor, pois auxilia no aumento da autoestima, além da mulher ter apoio familiar e ser mais valorizada psicologicamente. Pelo contrário, mau relacionamento conjugal, baixa renda familiar e transtorno psiquiátrico no passado, tem grande influência no desencadeamento da depressão pós-parto ${ }^{(9)}$.

Quando o assunto é DPP, estima-se que entre 10 a $15 \%$ das mulheres sofrem com sintomas depressivos na gravidez e que esta patologia vem sendo desmerecida, dando maior valor aos momentos psicóticos por causa de necessidade de internação em consequência de urgência. É necessário um olhar holístico na mãe e no recém-nascido (RN) para que seja feito os devidos encaminhamentos ${ }^{(6)}$.

No cenário mundial, a depressão configura-se como a quinta causa de morbimortalidade, sendo o sexo feminino o mais atingido. Nesse caso a atenção primária tem função essencial para prevenção, pois configura- se como porta de entrada para o SUS ${ }^{(4)}$.

A enfermagem já tem suas funções pré-definidas teoricamente, que se baseiam entre gestão da equipe multidisciplinar e APS, busca ativa de mulheres faltosas quando em acompanhamento, além de conhecimento sobre a patologia descri$\mathrm{ta}^{(4)}$.O esperado quanto à DPP é bem nítido, e dentre as atribuições do enfermeiro que se encontram na lei 1498/86, que vem a dizer sobre a assistência de enfermagem a gestante, parturiente e puérpera, o que não se sabe é se realmente isso vem a ser realizado na prática, mas não existe uma política pública direcionada diretamente com a DPP(10).

Visto que há uma escassez de pesquisas especificas de DPP, esta pesquisa vem buscar como os enfermeiros da atenção básica em um município do Centro Oeste mineiro lidam com o dia a dia da vivência entre diagnóstico e acompanhamento de mulheres com depressão pós-parto. A pesquisa objetivou analisar a percepção de enfermeiros sobre o diagnóstico, acompanhamento, tratamento e alta de mulheres portadoras de DPP no município de Divinópolis- MG.

\section{MÉTODOS}

Estudo de abordagem qualitativa e caráter descritivo. Neste tipo de estudo o 
pesquisador precisa estar envolvido em todas as etapas de construção do trabaIho a ser desenvolvido, a fim da busca da compreensão de experiências vividas por pessoas que se inserem no tema proposto, a partir da análise de suas falas, bem como da diversidade lógica de suas respostas, para que assim possa esta narrativa ser contextualizada de forma precisa e explicita, de maneira coerente, no qual o estudo seja de importante relevância para a sociedade ${ }^{(11)}$.

A pesquisa foi realizada no município de Divinópolis-MG, com os enfermeiros que compõem as Equipes de Estratégia de Saúde da Família (ESF) desta cidade. Os dados foram coletados em ESF's, no período de setembro de 2018. As ESF's foram definidas através de sorteios aleatórios em um total de dez.

A visita ocorreu em dias distintos, onde o roteiro foi deixado em algumas unidades para que respondessem de acordo com sua disponibilidade, foi recoIhido depois de respondido, outros foram gravados na hora da entrevista marcada. As informações foram angariadas e foi utilizado o método de saturação teórica, no qual houve a suspensão da inclusão de novos participantes quando atingiu nove entrevistados, pois a coleta de dados apresentou redundância ou repetição na avaliação do pesquisador.

Foi apresentado aos enfermeiros, pelos pesquisadores, um termo de consentimento livre e esclarecido (TCLE), assegurando-os de todos seus direitos sobre a pesquisa, tais como: sua confidencialidade, privacidade, proteção da imagem, assim como sua dignidade e autonomia de permanecer na pesquisa ou não. Os participantes foram totalmente esclarecidos sobre o objetivo da pesquisa e isentos de qualquer risco e possíveis incômodos, acarretados durante a mesma. Do mesmo modo, serão beneficiados com o resultado final, podendo trazer grandes avanços para sua atuação na comunidade.

Os dados de seis enfermeiros foram respondidos via escrita, três foram gravados, um não devolveu a pesquisa, por- tanto não contém recortes de "enf 6", as perguntas foram respondidas de acordo com a melhor forma que o enfermeiro encontrou; gravado ou escrito. Neste roteiro contém os dados referentes ao: conhecimento quanto à temática depressão pós-parto, tais como: como é o fluxo de atendimento à mulher com DPP, se o atendimento é de acordo com o princípio de integralidade do SUS, se o tratamento é humanizado e de qualidade, os pontos negativos e positivos no âmbito municipal quanto a DPP, como é realizada a capacitação dos profissionais, a importância da família no tratamento da DPP, se ocorrer abandono do tratamento como é realizada a busca ativa e se é feito tratamento da mulher e de seus familiares na unidade. $\mathrm{E}$ dados individuais do profissional, tais como: idade, unidade de trabalho, tempo de formação acadêmica, tempo de atuação na área de saúde pública.

Logo após o término das entrevistas os dados foram transcritos na íntegra, e os enfermeiros foram identificados pelas siglas Enf. 1 (enfermeiro 1...); Enf. 2 (enfermeiro 2) e assim sucessivamente, até atingir o número de saturação teórica dos dados.

A pesquisa atingiu saturação teórica quando coletados nove questionários (no qual ocorre suspensão da inclusão de novos participantes quando a coleta de dados passarem a apresentar redundância ou repetição na avaliação do pesquisador $^{(12)}$. A idade dos entrevistados varia entre 30 e 46 anos de idade e entre 1 ano e oito meses a 13 anos de prestação de serviço à saúde pública. Após organização das falas e análise das mesmas, para melhor compreensão dos dados foi necessário subdividir a pesquisa em duas categorias temáticas: gestão geral da atenção e integralidade da assistência.

Foi definido como critérios de inclusão à pesquisa: Ser enfermeiro, vinculado a ESF e aceitar participar da pesquisa, e como critérios de exclusão: Estar gozando férias ou licença no momento do estudo ou não querer participar do estudo.

O referencial utilizado foi o de Minayo que se subdivide em etapas, sendo elas: ordenação, que se dá pela organização do material coletado, transcrição e organização das falas coletadas, classificação do material e comparação das falas coletadas como é realmente, trazendo relevância para o trabalho a partir de fundamentos científicos e a análise propriamente dita ${ }^{(11)}$. O presente estudo obedeceu à resolução CNS/CONEP 466 de 12 de dezembro de 2012 que aprova as diretrizes e normas de pesquisas envolvendo seres humanos, aprovado com número de CAAE: 92058418.8.0000.5115.

\section{RESULTADOS}

Para melhor análise e compreensão, os dados foram divididos em duas categorias:

\section{Gestão da atenção primária}

Os profissionais da enfermagem das ESF'S ficam sem suporte literário pré-definido para seguir, caso de deparem com uma mulher com depressão pós-parto (NO).

Apesar de não conter um fluxograma desenhado, é notável que o caminho percorrido nas unidades seja padrão: começa pelo acolhimento, consulta de enfermagem a mulher e puericultura da criança, todos eles direcionam para o psicólogo, para o médico que prescreve medicação, ou a um psiquiatra, num encaminhamento secundário (NO):

Qualquer profissional da equipe pode acolher [...] (Clínico ou obstetra) da própria unidade ou do Sersan (nível secundário) (enf. 1). Atendida/acolhida [...] encaminhamos para serviço de psicologia (enf. 2)

Não há um fluxo definido na unidade [...] podem ser identificados através da consulta de enfermagem[...] provavelmente essa muIher vai precisar de medicação (enf. 3)

$\mathrm{Na}$ consulta de enfermagem eu identifico que uma mulher possa estar com depressão (enf. 4) 
Encaminhado para avaliação (enf.5) A puérpera/nutriz, atendida na unidade é acolhida para os primeiros atendimentos no pós-parto, a paciente que apresenta depressão é encaminhada ao psicólogo da unidade (enf. 8).

Não há um fluxo definido na unidade. A gestante que chega na unidade com sintomas é percebido pelo ACS em visita e encaminhada para consultas de enfermagem e se necessário, para psicóloga [...] se necessário (enf. 9).

Não tem um fluxo desenhado aqui na minha unidade, e eu também desconheço esse fluxo no município [...] qualquer alteração da emoção da mãe, tentamos integrar outros profissionais (enf. 10)

Percebe- se a falta de comunicação entre a gestão municipal e a ESF, que se descreve através da ausência de capacitação dos profissionais, e uma dificuldade de acesso ao serviço de saúde quando se precisa de uma contra referência, ou serviço de secundário (NO).

Falta de qualificação de muitos profissionais para diagnosticar [...] dificuldade de acesso no atendimento em outros setores no nível secundário (enf. 1).

Falta muita sensibilização dos profissionais para atuarem identificando a evolução pós-parto [...] (enf. 3)

Nós não temos essa contra referência (enf. 4)

A comunicação entre serviços afins [...] falta de padronização (enf. 5)

Menor aproximação de referência técnica de saúde da mulher (enf. 7)

Falta de capacitação (enf. 8)

Demora do agendamento em outras unidades (enf. 9)

Nessa unidade nunca foi comentado, a nível de estratégia, treina- mento e capacitação não conheço [...] poderiam conversar mais sobre isso (enf. 10)

No mesmo assunto, mas abordado de forma positiva, houve várias divergências entre as conclusões dos profissionais. Entende- se que o município falta com a estratégia a ponto de que, de acordo com os recortes, o ponto positivo apontado é a presença do profissional enfermeiro diretamente ligado com a mulher que precisa do serviço, ao contrário do intuito buscado, somente foi reforçado um lado negativo (NO).

Quantidade razoável de profissionais para o atendimento aos usuários (enf. 1)

Existem profissionais muito bons (enf. 3)

Eu não acho que tem ponto positivo nenhum [...] educação continuada, de nada [...] nesses seis anos que eu estou na prefeitura nunca tive nenhum treinamento a respeito disso [...] Eles não dão suporte nenhum (enf. 4)

Fica difícil falar de sistema a nível municipal (enf. 5)

Não tem (enf. 9)

Presença do enfermeiro que está diretamente com essa mulher [...] manter, em cada unidade a lotação do profissional enfermeiro (enf. 10)

Apesar de várias falhas do sistema, o empenho das equipes está presente nas unidades, estas têm por base, o acolhimento, e a necessidade de mostrar para a mulher a preocupação com ela, tendo no mínimo mesmo que demore um profissional que possa encaminhar (NO).

Encaminha diretamente ao psicólogo (enf. 1)

Apesar de todas as dificuldades que temos na unidade conseguimos acompanhar esta mulher (enf. 2)
A questão do acolhimento é necessário [...] ouvir as suas demandas (enf. 3)

Nós conversamos [...] o apoio [...] sempre estamos com as portas abertas para dar o suporte [...] chamar para o consultório, conversar, atender e ouvir (enf. 4)

O paciente é encaminhado ao serviço de referência (psicologia) (enf. 8)

A mulher é acolhida, acompanhada pela equipe na unidade e domicílio (enf. 9)

Atender a mulher como um todo [...] tem o profissional pra acolher aqui (enf.10)

Neste, há um destaque de empenho de um enfermeiro, que é motivado a ponto de sugestão da participação de profissionais na criação de um fluxo.

Deveríamos fazer um fluxo e apresentar ao município para maior divulgação (enf. 9).

Apesar do empenho dos profissionais para atenderem as mulheres, os enfermeiros se mostraram receosos quando o assunto abordado (NO).

Infelizmente, a unidade não está preparada para lidar com a DPP e não se prepara para isso (enf. 1) A unidade mesmo nunca fez nenhuma ação nesse sentido (enf. 2)

Esse é um ponto realmente fraco [...] não estamos preparados para lidar com isso [...] assunto pouco discutido [...] falta da capacitação e educação permanente (enf. 3)

Eu nem sei se estamos muito preparados [...] a secretaria também não dá muito suporte [...] acaba que fica subnotificado (enf. 4)

Devemos abordar mais sobre o tema (enf. 7)

Orientações no dia dia (enf. 8)

Não há uma capacitação para preparar os profissionais [...] está 
relacionado ao paciente (enf. 9) Prepara a equipe no sentido de ouvir (enf. 10)

A ESF conta com o profissional ACS, que tem como uma das suas funções, ser a ligação à mulher e a unidade, nesse caso quando mulheres ficam faltosas, fica a cargo do agente de saúde fazer a busca ativa. Percebe-se que além da ACS também se usa muito o contato telefônico para chamada de faltosas, apesar de algumas unidades não usufruírem do serviço do ACS (NO).

Não é realizada busca ativa (enf. 1) Telefone e quando possível visita domiciliar (enf. 2)

Tentamos fazer contato telefônico [...] tentamos ir a casa (enf. 3) Pedimos para ACS estar indo na casa da paciente para procurar saber o que aconteceu, porque faltou [...] reagenda a consulta pra ela estar voltando (enf. 4)

ACS [...] vai a casa do paciente (enf. 5)

Fazemos busca ativa domiciliar através do ACS, e a equipe realiza visita de acordo com a demanda (enf. 7)

A busca ativa é feita (enf. 8)

Realizada pelo ACS (enf. 9)

ACS [...] telefone corporativo (enf. 10)

Integralidade da assistência

É possível perceber que as unidades contrapõem esses conceitos, as ESF’s não estão preparadas para um atendimento integral, não conseguem atingir este proposto, podendo este ser relacionado com o modelo de gestão adotado, pela pouca demanda existente de DPP, ou pela falta de capacitação, ou seja, isso implica diretamente no usuário, que acaba tendo um atendimento fracionado (NO).

Apesar de a maioria das unidades não conseguir um atendimento completo, existe um destaque de seis que, vem a mostrar que apesar da falta de percurso predefinido a seguir, elas conseguem atender de forma mais completa a puérpera, em relação às outras, mas, ainda assim não conseguem atingir por inteiro o conceito de integralidade.

Infelizmente, na maioria dos casos, o atendimento ocorre de forma fragmentada (Enf. 1)

Temos discussão (enf. 2)

É um atendimento fragmentado (Enf. 3)

No caso de a mulher precisar de um psiquiatra acaba que fraciona [...] não tem uma contra referência depois para ver como está sendo o acompanhamento dela (Enf. 4)

Discussão dos casos (enf. 5)

Tentam atender e suprir todas as demandas (Enf. 8)

$\mathrm{O}$ atendimento domiciliar ou na unidade [...] Reuniões a cada 15 dias, pra discussão de casos importantes (enf. 7)

Equipe tem uma discussão do caso (enf. 9)

Além de contra referenciar [...] está discutindo esses casos (enf. 10)

O acolhimento do usuário está diretamente interligado ao processo de humanização e pode interferir diretamente no tratamento ao paciente, uma vez estando em um ambiente acolhedor o mesmo se sentirá confortável para expor seus problemas e preocupações, o que resultará em uma melhor resolução. Em recortes das entrevistadas, pode se perceber que há uma grande preocupação em fazer um bom acolhimento, buscando a criação de vínculo com as pacientes, transmitindo Ihes confiança, apoio e ajuda (NO).

Acolhemos cada usuário em sua demanda através da escuta (enf. 1) Acolhimento humanizado [...] a escuta (enf.2)

Acolhimento faz toda diferença (Enf. 3)

Procuramos sempre acolher bem [...] escutando, apoiando, dando importância para o que a paciente fala (Enf. 4)

Ausculta qualificada [...] o cliente se sente acolhido e respeitado (enf.5)

Forma mais acolhedora: utilizamos uma escuta qualificada (Enf. 7)

A equipe é orientada quanto o acolhimento (Enf. 8)

É criado um vínculo, bom atendimento e confiança (Enf. 9)

Procuramos integrar na criação de vínculo (Enf. 10).

Além de uma equipe preparada para lidar com a DPP, tem- se grande importância que a família esteja inserida no tratamento dessa mulher, pois de acordo com os recortes, enfermeiros veem a família com grande valor na inserção e apoio no tratamento da mulher (NO).

É fundamental o auxílio familiar no tratamento da DPP, principalmente do parceiro [...] a equipe de saúde sozinha e os medicamentos ficam difícil (enf. 1)

Base estrutural e apoio (enf. 2)

A família é o mais importante depois da mulher [...] muitas vezes a família adoece junto [...] preciso ser acolhida junto com mulher (enf. 3)

Família é primordial (enf. 5)

É de extrema importância (enf. 7) Apoio familiar ajuda no processo de aceitação, e também na redução dos danos (enf. 8)

O tratamento adequado da DPP está relacionado... Integridade física, mental e harmonia da família (enf. 9)

A família que vai dar um suporte em casa e até um feed back para nós profissionais(enf. 10)

Percebe- se o esforço das unidades em adicionar a família no tratamento da mulher, e quando essa família ou algum integrante dela passa a interagir com a patologia de forma intensa e que possa vir a 
precisar de tratamento, a unidade mostra seu esforço em dar continuidade a um tratamento também para a família (NO).

Fortalecimento da rede familiar e na psicologia terapia (enf. 2)

Acolher a família junto com a mulher (enf. 3)

Orienta também passar pelo psicólogo (enf. 4)

Solicitamos ao serviço de psicologia (enf. 5)

Reunimos entre os profissionais da equipe e decidimos uma meIhor estratégia de abordagem da família (enf. 7)

Aconselhamento psicológico para a família (enf. 8)

Acompanhamento da família e do companheiro [...] acompanhamento psicológico da família (enf. 9)

Da mesma forma que essa puérpera [...] podemos acolher esse familiar [...] as vezes não damos a real importância que a família tem (enf.10)

\section{DISCUSSÃO}

Entre um recorte e outro é possível perceber que o ponto ápice dessa pesquisa gira em torno da falta de capacitação dos profissionais quanto a DPP, problema que atinge diretamente um possível diagnóstico, que pode gerar subnotificações da patologia e a presença de um fracionamento da assistência, que danifica totalmente a prestação de serviços a mulher, esta que por sua vez precisa de integralidade na assistência prestada.

Percebe-se que há um grande ponto falho do sistema municipal ao assessoramento das unidades em relação à DPP. Visto que, o município tem papel primordial no seguimento da saúde pública, fazendo pactos intermunicipais, aplicação de políticas públicas e se necessário parte da criação dessas políticas ${ }^{(13)}$ e isto se aplica diretamente a depressão pós-parto, já que se configura uma doença de saúde pública ${ }^{(3)}$

\section{Entre um recorte} e outro é possível perceber que o ponto ápice dessa pesquisa gira em torno da falta de capacitação dos profissionais quanto a DPP, problema que atinge diretamente um possível diagnóstico, que pode gerar subnotificações da patologia e a presença de um fracionamento da assistência, que danifica totalmente a prestação de serviços a mulher, esta que por sua vez precisa de integralidade na assistência prestada.
A busca ativa é uma expressão muito usada pelos sistemas vigilantes de saúde e é caracterizada por uma forma de buscar pessoas com a finalidade de detectar uma sintomatologia. Por meio desta, é possível se relacionar com o paciente e compreender toda sua interação com o mundo que o cerca. Desta forma também promove conhecer seu estado mental e de sua família, suas condições de bem-estar, como também examinar se existe mais de uma patologia correlacionada. Na DDP a busca ativa é um instrumento fundamental, pois devido ao estado emocional em que se encontra a puérpera, ela pode se sentir desestimulada a dar continuidade ao tratamento, sendo de extrema importância neste momento uma reorientação ${ }^{(15)}$.

O profissional de Enfermagem atua como supervisor da atenção primária de saúde, este quando motivado a planejar uma assistência voltada ao cuidado, deve se atentar para a integralidade em uma esfera individual, focando no âmbito coletivo entre equipe de enfermagem e serviços de Saúde. Para isto é necessário associar as diversas classes profissionais, referenciando e contra referenciando os usuários no intuito de dar seguimento aos cuidados em uma rede de atenção ${ }^{(16)}$.

Para a Política Nacional De Humanização (PNH) um elemento fundamental para humanização é o ambiente, caracterizado por um local onde há uma relação efetiva entre os sujeitos sendo instrumento favorável para o procedimento de trabalho, assegurando a privacidade e a identidade do ser humano. Para que este processo seja efetuado, é imprescindível que todos estes fatores sejam executados de forma ética legal e moral ${ }^{(16)}$.

A harmonia coletiva e familiar passa por transformações prejudiciais após o aparecimento da DPP e se torna complexo para a mulher manifestar suas emoções, uma vez que as mesmas veem obstáculos facilmente, tornando-se mais difícil quando há a falta de compreensão por parte do cônjuge ${ }^{(17)}$. A relação com os demais filhos se tor- 
na fragmentada, pois passam a serem cuidados por pessoas distintas, há ainda sentimentos de reprovação e discriminação ${ }^{(18)}$. Sendo assim fica evidente que a família é fundamental na vida da mulher que sofre de DPP, pois o comportamento e as reações que a mesma desempenha em relação a ela pode remeter satisfatoriamente ou prejudicialmente em seu quadro clínico.

Dentre as limitações encontradas para realização do estudo, destaca-se a dificuldade por parte dos enfermeiros responderem o questionário devido a alta demanda da unidade.

\section{CONCLUSÃO}

Este trabalho traz a importância de ter um assessoramento à nível de gestão em relação a saúde pública, e enfatiza que mesmo não tendo apoio do nível municipal diretamente nesse assunto, as equipes se empenham continuamente para a melhoria da saúde pública e, mesmo que não estejam preparadas para um diagnóstico e acompanhamento de mulheres que tem DPP as unidades se mostram bastante acolhedoras. É fornecido um ambiente confortável e confiável para as pacientes, estabelecendo vínculo forte com a mulher e a família, contexto predominantemente importante da saúde públi- ca que não somente na depressão pós-parto, é a base da confiança do usuário no sistema que foi criado pra servir a população.

A pesquisa possui grande valor acadêmico, pois possibilitou um melhor entendimento da atenção básica relacionado à mulher, que vai além dos conhecimentos adquiridos através das grades curriculares. Deixa- se explícito um alerta a saúde pública de Divinópolis, que precisa buscar rigorosamente uma melhoria no sistema de saúde da mulher relacionado a DPP, contribuindo assim para um atendimento integral que vai de acordo com as diretrizes do Sistema Único de Saúde.

\section{Referências}

1. Campos RTO, Ferrer AL, Gama CAP, Campos GWS, Trapé TL, Dantas DV. Avaliação da qualidade do acesso na atenção primária de uma grande cidade brasileira na perspectiva dos usuários. Rev. Saúde debate. 2014; 38(spe):252-264. Disponível em: https://www.scielo.br/scielo.php?pi$\mathrm{d}=$ S0103-11042014000600252\&script=sci_abstract\&tlng=pt. Acesso em 23 jul. 2020. Doi: https://doi.org/10.5935/0103-1104.2014S019.

2. Fontana KC, Lacerda JT, Machado PMO. 0 processo de trabalho na atenção básica à saúde: Avaliação da gestão. Rev. Saúde debate. 2016; 40(110):6480. Disponivel em: https://www.scielo.br/scielo.php?script=sci_arttext\&pi$d=S 0103-11042016000300064 \&$ lng=pt\&tlng=pt. Acesso em 23 jul. 2020. Doi: https://doi.org/10.1590/0103-1104201611005.

3. Brocchi BS, Bussab VSR, David V. Depressão pósparto e habilidades pragmáticas: comparação entre gêneros de uma população brasileira de baixa renda. Audiol Commun Rev. São Paulo. 2015; 20(3):262-8. Disponível em: https://www.scielo.br/scielo.php?pid=\$2317=64312015000300262-\&scriptsci_abstract\&tlng=pt. Acesso em 23 jul. 2020. Doi: https://doi.org/10.1590/ 2317-6431-ACR-2015-1538.

4. Gonçalves FBAC, Almeida MC. Atuação da enfermagem frente à depressão pós-parto. Ensaios e Ciênc. 2019; 23(2):140-147. Disponível em: https://revista.pgsskroton.com/index.php/ensaioeciencia/article/view/6655. Acesso em 23 jul. 2020. Doi: https://doi.org/10.17921/1415-6938.2019v23n2p140-147.

5. Nader JM, Moreira NC, Carvalho LOO, Rassi A, Brito AFP, Silveira MMM. Correlação entre autoeficácia em amamentação e depressão pós-parto. Braz. J. Hea. Rev. 2020; 3(2):3875-3888. Disponível em: https://www.brazilianjournals. com/index.php/BJHR/article/view/9422. Acesso em 23 jul. 2020. Doi: https:// doi.org/10.34119/bjhrv3n2-210.

6. Lima MOP, Tsunechiro MA, Bonadio IC, Murata M. sintomas depressivos na gestação e fatores associados: estudo longitudinal. Acta paul. enferm. 2017; 30 (1): 39-46. Disponível em: https://www.scielo.br/scielo.php?script=sci_arttext\&pid=S0103-21002017000100039\&lng=en. Acesso em 23 jul. 2020. Doi: https://doi.org/10.1590/1982-0194201700007.

7. Hartmann JM, Mendoza-sassi RA, Cesar JA. Depressão entre puérperas: prevalência e fatores associados. Cad. Saúde Pública 2017; 33(9):e00094016. Disponivel em: https://www.scielo.br/pdf/csp/v33n9/1678-4464-csp-3309-e00094016.pdf. Acesso em 23 jul. 2020. Doi: https://doi.org/10.1590/ 0102-311x00094016.

8. Morais MLS, Fonseca LAM, David VF, Viegas LM, Otta E. Fatores psicossociais e sócio demográficos associados à depressão pós-parto: Um estudo em hospitais público e privado da cidade de São Paulo, Brasil. Estudos de Psicologia. 2015; 20(1):40-9.Disponível em: https://www.scielo.br/pdf/epsic/v20n1/ 1413-294X-epsic-20-01-0040.pdf. Acesso em 23 jul. 2020. Doi: https://doi. org/10.5935/1678-4669.20150006.
9. Pedrotti, Bruna Gabriella, \& Frizzo, Giana Bitencourt. (2019). Influência da chegada do bebê na relação conjugal no contexto de depressão pós-parto: perspectiva materna. Pensando familias, 23(1):73-88. Disponível em: http://pepsic.bvsalud.org/scielo.php?script=sci_abstract\&pi$d=S 1679-494 X 2019000100007 \&$ lng=pt\&nrm=iso. Acesso em 23 jul. 2020.

10. Brasil. Lei $n$ 7.498/86, de 25 de junho de 1986. Dispõe sobre a regulamentação do exercício da Enfermagem e dá outras providências. Diário oficial da união. 26 de junho de 1986.

11. Minayo MCS. Análise qualitativa: teoria, passos e fidedignidade. Ciência \& Saúde Coletiva. 2018; 17(3)621-626, 2012. Disponível em: https://www.scielo. br/scielo.php?pid=S1413-81232012000300007\&script=sci_abstract\&tlng=pt. Acesso em 24 abr. Doi: https://doi.org/10.1590/S1413-81232012000300007. 12. Fontanella BJB, Júnior RM. Saturação teórica em pesquisas qualitativas: contribuições psicanalíticas. Psicologia em Estudo. 2012; 17(1):63-71.Disponível em: https://www.scielo.br/pdf/pe/v17n1/v17n1a07.pdf. Acesso em 23 jul. 2020. 13. Brasil. Lei $n 8080$ de 19 de setembro de 1990. Dispõe sobre as condições para a promoção, proteção e recuperação da saúde, a organização e o funcionamento dos serviços correspondentes e da outras providências. Diário oficial da união. 20 de setembro de 1990.

14. Meira BM, Pereira PAS, Silveira MFA, Gualda DMR, Santos JHPO. Desafios para profissionais da atenção primária no cuidado à mulher com depressão pós-parto. Rev. Texto contexto enfermagem. 2015; 24(3):706-12. Doisponível em: https://www.scielo.br/scielo.php?script=sci_arttext\&pi$d=S 0104-07072015000300706 \& \operatorname{lng}=e n \& t / n g=e n$. Acesso em 23 jul. 2020. Doi: https://doi.org/10.1590/0104-0707201500049-14.

15. Pereira MO, Amorim A, Vidal V, Falavigna MF, Oliveira MAF. Busca ativa para conhecer o motivo da evasão de usuários em serviço de saúde mental. Rev. Acta paulista de enfermagem. 2013; 26(5):409-12. Disponível em: https:// www.scielo.br/scielo.php?pid=S0103-21002013000500002\&script=sci_abstract\&tlng=pt. Acesso em 23 jul. 2020. Doi: https://doi.org/10.1590/S010321002013000500002.

16. Chaves LDP, Mininel VA, Silva JAIM, Alves LR, Silva MF, Camelo SHH. Supervisão de enfermagem para a integralidade do cuidado. Revista Brasileira de Enfermagem. 2017; 70(5):1106-11. Disponível em: https://www.scielo.br/ scielo.php?pid=S0034-71672017000501106\&script=sci_arttext\&tlng=pt. Acesso em 23 jul. 2020. Doi: https://doi.org/10.1590/0034-7167-2016-0491.

17. Melo SW, Carolina JC, Flávia PMM, Francisco ASV. Relacionamento familiar, necessidades e convivência social de mulheres com depressão pós-parto. Rev. revista de enfermagem. 2015; 9(3): 7065-70.

18. Rodrigues WLC, Branco JGO, Facundo SHBC, Costa FBC, Oliveira CJ. Consequências da depressão pós-parto no desenvolvimento infantil: revisão integrativa. Nursing (Säo Paulo). 2019; 22(250):2728-2733. 